\section{THU0174 CHARACTERIZATION OF REMISSION IN PATIENTS WITH RHEUMATOID ARTHRITIS TREATED WITH UPADACITINIB OR COMPARATORS}

Stephen Hall ${ }^{1}$, Tsutomu Takeuchi ${ }^{2}$, Glen Thomson ${ }^{3}$, Paul Emery ${ }^{4}$ Bernard Combe $^{5}$, Andrea Everding ${ }^{6}$, Karel Pavelka ${ }^{7}$, Yanna Song ${ }^{8}$, Tim Shaw ${ }^{8}$, Alan Friedman ${ }^{8}$, In-Ho Song ${ }^{8}$, Eduardo Mysler ${ }^{9}{ }^{1}$ Monash Univ, Cabrini Health and Emeritus Research, Malvern, Australia; ${ }^{2}$ Keio Univ School of Medicine, Tokyo, Japan; ${ }^{3}$ CIADS Research, Winnipeg, Canada; ${ }^{4}$ Leeds Inst of Rheumatic and Musculoskeletal Medicine, Leeds NIHR BRC, Leeds, United Kingdom, ${ }^{5} \mathrm{CHU}$ Montpellier, Univ Montpellier, Montpellier, France; ${ }^{6} \mathrm{HRF}$ Hamburger Rheuma Forschungszentrum, Hamburg, Germany; ${ }^{7}$ Charles Univ, Prague, Czech Republic ${ }^{8}$ AbbVie Inc, North Chicago, United States of America; ${ }^{9}$ Organización Medica de Investigación, Buenos Aires, Argentina

Background: Across all phase 3 studies, treatment with upadacitinib (UPA), a JAK1-selective inhibitor, was associated with significantly higher remission (REM) rates, compared to placebo (PBO) or active comparators, in RA patients (pts) who were methotrexate (MTX)-naive, had inadequate response to conventional synthetic (csDMARD-IR) or had inadequate response or intolerance to biologic DMARDs (bDMARD-IR) Objectives: REM definitions are based on composite scores of various individual assessments of disease activity. To determine the response to UPA on REM and component assessments, we assessed the proportions of pts achieving REM using multiple REM definitions, and the improvement in their respective individual components, compared to PBO or active comparators, in 3 different RA pt populations spanning a range of RA pt populations. Methods: Three phase 3 studies included pts who were MTX naïve (SELECT EARLY, $\mathrm{n}=945$ ), MTX-IR (SELECT COMPARE, $\mathrm{n}=1629$ ) and bDMARD-IR (SELECT BEYOND, $\mathrm{n}=498$ ). The proportion of $\mathrm{pts}$ achieving REM at Week (Wk) 12 by 4 definitions (DAS28-CRP<2.6; CDAI $\leq 2.8$; SDAl $\leq 3.3$ and Boolean, defined as $\leq 1$ for TJC, SJC, patient's global assessment of disease activity [PtGA], and CRP $\leq 1 \mathrm{mg} / \mathrm{L}$ ) were determined. For each definition of REM, the mean change in each of the respective component scores was also assessed. Binary endpoints are based on Non-responder imputation (NRI), and continuous endpoints on mixed-effect model repeat measurement (MMRM). Comparisons were made between UPA-treated groups vs respective control arms (MTX, adalimumab [ADA] or PBO).

Results: Pt demographics and disease characteristics have been previously reported. ${ }^{1-3}$ At 12 wks, in EARLY and COMPARE, a significantly greater proportion of pts receiving UPA $15 \mathrm{mg}$ or $30 \mathrm{mg}$ QD achieved REM by all 4 definitions vs MTX, PBO or ADA (Table). In BEYOND, (a refractory population many of whom had inadequate response to multiple bDMARDs), a significantly greater proportion of pts receiving UPA $30 \mathrm{mg}$ achieved all REM definitions vs PBO within the first 12 wks, with significantly greater proportions on UPA $15 \mathrm{mg}$ achieving DAS28-CRP $<2.6$ and Boolean REM (Table). Rates of REM in BEYOND further increased through Wk 24 for both dose groups. ${ }^{1}$ Compared to respective control groups, pts receiving UPA 15 or $30 \mathrm{mg}$ QD had significantly greater improvements in each REM disease component (except for PhGA vs ADA in COMPARE). Significantly more pts receiving UPA also achieved the required cutoffs on the individual components of Boolean REM compared to respective controls. Conclusion: Significantly greater proportions of pts receiving UPA 15 or $30 \mathrm{mg}$ achieved REM by multiple definitions at 12 wks compared to PBO, MTX or ADA. All disease activity components of each REM definition were significantly improved in pts receiving UPA compared to MTX or $\mathrm{PBO}$, and all Boolean components were significantly improved in pts receiving UPA $15 \mathrm{mg}$ compared to ADA.

\section{REFERENCES :}

[1] Genovese, et al. 2018;Lancet.18;31116-4.

[2] van Vollenhoven R, et al. Arthritis Rheumatol. 2018;70 (supp10).

[3] Fleischmann, et al. Arthritis Rheumatol. 2018;70 (supp10).

Acknowledgement: AbbVie, Inc was the study sponsor, contributed to study design, data collection, analysis \& interpretation, and to writing, reviewing, and approval of final version. Medical writing support was provided by Naina Barretto, $\mathrm{PhD}$, of AbbVie, Inc.

Disclosure of Interests: Stephen Hall Grant/research support from: AbbVie Inc, BMS, Lilly, Janssen, Pfizer, UCB, and Novartis, Consultant for: AbbVie Inc, BMS, Lilly, Janssen, Pfizer, UCB, and Novartis, Tsutomu Takeuchi Grant/research support from: Astellas Pharma Inc, Chugai Pharmaceutical Co, Ltd., Daiichi Sankyo Co., Ltd., Takeda Pharmaceutical Co., Ltd., AbbVie GK, Asahikasei Pharma Corp., Mitsubishi Tanabe Pharma Co., Pfizer Japan Inc., Eisai Co., Ltd., AYUMI Pharmaceutical Corporation, Nipponkayaku Co. Ltd., Novartis Pharma K.K., Grant/research support from: AbbVie, Asahi Kasei, Astellas, AstraZeneca, AYUMI, Bristol-Myers Squibb, Chugai, Daiichi Sankyo, Eisai, Eli Lilly Japan, Janssen, Mitsubishi Tanabe, Nippon Kayaku, Novartis, Pfizer Japan Inc, Taiho, Taisho Toyama, Takeda, Teijin, Grant/ research support from: Astellas Pharma Inc., Bristol Myers Squibb, Chugai Pharmaceutical Co., Ltd., Mitsubishi Tanabe Pharma Co., Pfizer Japan Inc. Santen Pharmaceutical Co., Ltd., Takeda Pharmaceutical Co., Ltd., Teijin Pharma Ltd., AbbVie GK, Asahi Kasei Pharma Corp., Taisho Toyama Pharmaceutical Co., Ltd., SymBio Pharmaceuticals Ltd., Janssen Pharmaceutical K.K., Celltrion Inc., Nipponkayaku Co. Ltd., and UCB Japan, Consultant for: Astra Zeneca K.K., Eli Lilly Japan K.K., Novartis Pharma K.K., Mitsubishi Tanabe Pharma Co., Abbivie GK, Nipponkayaku Co.Ltd, Janssen Pharmaceutical K.K., Astellas Pharma Inc., Taiho Pharmaceutical Co. Ltd., Chugai Pharmaceutical Co. Ltd., Taisho Toyama Pharmaceutical Co. Ltd. GlaxoSmithKline K.K., UCB Japan Co. Ltd., Consultant for: AbbVie, Asahi Kasei, Astellas, AstraZeneca, AYUMI, Bristol-Myers Squibb, Chugai, Daiichi Sankyo, Eisai, Eli Lilly Japan, Janssen, Mitsubishi Tanabe, Nippon Kayaku, Novartis, Pfizer Japan Inc, Taiho, Taisho Toyama, Takeda, Teijin, Consultant for: Astra Zeneca K.K., Eli Lilly Japan K.K., Novartis Pharma K.K., Mitsubishi Tanabe Pharma Co., Asahi Kasei Medical K.K., AbbVie GK, Daiichi Sankyo Co., Ltd., Bristol Myers Squibb, and Nipponkayaku Co. Ltd., Speakers bureau: Astellas Pharma Inc., Bristol Myers Squibb, Chugai Pharmaceutical Co., Ltd., Mitsubishi Tanabe Pharma Co., Pfizer Japan Inc., Santen Pharmaceutical Co., Ltd., Takeda Pharmaceutical Co., Ltd., Teijin Pharma Ltd., AbbVie GK, Asahi Kasei Pharma Corp., Taisho Toyama Pharmaceutical Co., Ltd., SymBio Pharmaceuticals Ltd., Janssen Pharmaceutical K.K., Celltrion Inc., Nipponkayaku Co. Ltd., and UCB Japan, Speakers bureau: AbbVie, Asahi Kasei, Astellas, AstraZeneca, AYUMI, Bristol-Myers Squibb, Chugai, Daiichi Sankyo, Eisai, Eli Lilly Japan, Janssen, Mitsubishi Tanabe, Nippon Kayaku, Novartis, Pfizer Japan Inc, Taiho, Taisho Toyama, Takeda, Teijin, Speakers bureau: AbbVie GK., Bristol-Myers K.K., Chugai Pharmaceutical Co. Ltd., Mitsubishi Tanabe Pharma Co., Pfizer Japan Inc., Astellas Pharma Inc, Diaichi Sankyo Co. Ltd., Eisai Co. Ltd., Sanofi K.K., Teijin Pharma Ltd., Takeda Pharmaceutical Co. Ltd., Novartis Pharma K.K., Glen Thomson Grant/research support from: AbbVie, Consultant for: Amgen, Paul Emery Grant/research support from: Pfizer, MSD, AbbVie, Bristol-Myers Squibb, Roche, Consultant for: Pfizer, MSD, AbbVie, Bristol-Myers Squibb, UCB, Roche, Novartis, Gilead,Samsung, Sandoz and Lilly, Bernard Combe Consultant for: Abbvie, Bristol-Myers Squibb, Gilead, Janssen, Eli Lilly, MSD Novartis, Pfizer, Roche-Chugai, Sanofi, UCB, Andrea Everding: None declared, Karel Pavelka: None declared, Yanna Song Shareholder of: AbbVie, Employee of: AbbVie, Tim Shaw Shareholder of: AbbVie Inc, Employee of: AbbVie Inc, Alan Friedman Shareholder of: AbbVie, Employee of: AbbVie, In-Ho Song Shareholder of: AbbVie Inc, Employee of: AbbVie Inc, Eduardo Mysler Grant/research support from: AbbVie, Bristol-Myers Squibb, Eli Lilly, Pfizer, Novartis, Janssen, Grant/research support from: AbbVie, Bristol-Myers Squibb, Eli Lily, Janssen, Medimmune, Pfizer Inc, and Roche Consultant for: AbbVie, Bristol-Myers Squibb, Eli Lily, Janssen, Medimmune, Pfizer Inc, and Roche, Speakers bureau: AbbVie, Bristol-Myers Squibb, El Lilly, Pfizer, Novartis, Janssen, Speakers bureau: AbbVie, Bristol-Myers Squibb, Eli Lily, Janssen, Medimmune, Pfizer Inc, and Roche

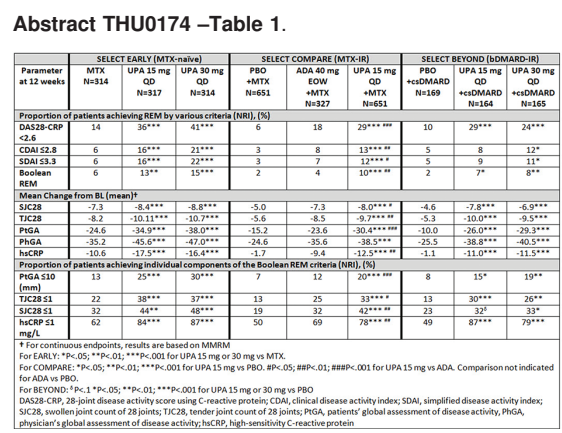

DOI: 10.1136/annrheumdis-2019-eular.2003

\section{THU0175 DOSE-DEPENDENT RISK OF METHOTREXATE FOR RENAL IMPAIRMENT IN PATIENTS WITH RHEUMATOID ARTHRITIS}

Keigo Hayashi, Kenei Sada, Yosuke Asano, Sumie Hiramatsu Asano, Yuriko Yamamura, Keiji Ohashi, Michiko Morishita, Haruki Watanabe,

Mariko Narazaki, Yoshinori Matsumoto, Tomoko Kawabata, Jun Wada. Okayama University Graduate School of Medicine, Dentistry and Pharmaceutical Sciences, Department of Nephrology, Rheumatology, Endocrinology and Metabolism, Okayama, Japan

Background: Methotrexate (MTX) is a mainstay in the therapy of rheumatoid arthritis (RA). It is recommended that MTX should be rapidly 